\title{
Effect of endurance versus resistance training on quadriceps muscle dysfunction in COPD: a pilot study
}

This article was published in the following Dove Press journal:

International Journal of COPD

27 October 2016

Number of times this article has been viewed

\section{Ulrik Winning lepsen' \\ Gregers Druedal Wibe \\ Munch' \\ Mette Rugbjerg' \\ Anders Rasmussen Rinnov' \\ Morten Zacho' \\ Stefan Peter Mortensen ${ }^{1,2}$ \\ Niels H Secher ${ }^{3}$ \\ Thomas Ringbaek ${ }^{4}$ \\ Bente Klarlund Pedersen' \\ Ylva Hellsten ${ }^{5}$ \\ Peter Lange ${ }^{1,4,6}$ \\ Pia Thaning ${ }^{1,4}$ \\ 'The Centre of Inflammation and Metabolism and the Centre for Physical Activity Research, \\ Rigshospitalet, University of Copenhagen, Denmark, ${ }^{2}$ Department of Cardiovascular and Renal Research, University of Southern Denmark, Odense, ${ }^{3}$ Department of Anesthesiology, University of Copenhagen, Rigshospitalet, Copenhagen, ${ }^{4}$ Department of Respiratory Medicine, University Hospital Hvidovre, Hvidovre, ${ }^{5}$ Department of Nutrition, Exercise and Sports, University of Copenhagen, ${ }^{6}$ Department of Public Health, Section of Social Medicine, University of Copenhagen, Copenhagen, Denmark}

Correspondence: Ulrik Winning lepsen The Centre of Inflammation and Metabolism and the Centre for Physical Activity Research, Rigshospitalet, University of Copenhagen, Blegdamsvej 9, 2100 Copenhagen, Denmark

Tel +4535457617

Fax +45 35457644

Email ulrik_winning@hotmail.com
Introduction: Exercise is an important countermeasure to limb muscle dysfunction in COPD. The two major training modalities in COPD rehabilitation, endurance training (ET) and resistance training (RT), may both be efficient in improving muscle strength, exercise capacity, and health-related quality of life, but the effects on quadriceps muscle characteristics have not been thoroughly described.

Methods: Thirty COPD patients (forced expiratory volume in 1 second: $56 \%$ of predicted, standard deviation [SD] 14) were randomized to 8 weeks of ET or RT. Vastus lateralis muscle biopsies were obtained before and after the training intervention to assess muscle morphology and metabolic and angiogenic factors. Symptom burden, exercise capacity (6-minute walking and cycle ergometer tests), and vascular function were also assessed.

Results: Both training modalities improved symptom burden and exercise capacity with no difference between the two groups. The mean (SD) proportion of glycolytic type IIa muscle fibers was reduced after ET (from $48 \%$ [SD 11] to $42 \%$ [SD 10], $P<0.05$ ), whereas there was no significant change in muscle fiber distribution with RT. There was no effect of either training modality on muscle capillarization, angiogenic factors, or vascular function. After ET the muscle protein content of phosphofructokinase was reduced $(P<0.05)$ and the citrate synthase content tended increase $(P=0.08)$ but no change was observed after RT.

Conclusion: Although both ET and RT improve symptoms and exercise capacity, ET induces a more oxidative quadriceps muscle phenotype, counteracting muscle dysfunction in COPD.

Keywords: muscle fibers, phosphofructokinase-1, vasodilation, rehabilitation, randomized controlled trial

\section{Introduction}

COPD is characterized by airflow limitation causing dyspnea, exercise intolerance, and limb muscle dysfunction. ${ }^{1}$ The beneficial effects of exercise training on disease burden, exercise capacity, and muscle function are well documented in patients with COPD. ${ }^{1-3}$ Endurance training (ET) has been the cornerstone in pulmonary rehabilitation; ${ }^{2}$ yet to prevent muscle weakness and atrophy, resistance training (RT) is also recommended. ${ }^{4,5}$ A meta-analysis comparing the effects of ET and RT in patients with COPD showed similar improvements in health-related quality of life, exercise capacity, and leg muscle strength. ${ }^{6}$ However, the beneficial effects of ET and RT are probably induced by different muscle adaptations ${ }^{7}$ and could counteract different pathological components of muscle dysfunction in COPD. Limb muscle dysfunction in COPD presents as muscle wasting, reduced strength, fiber-type shift from type I to type II, and reduced oxidative capacity. ${ }^{1}$ Although studies have explored the intrinsic muscular effects of both ET 
and RT, these studies compared COPD patients with healthy subjects in case-control designs. ${ }^{4,8-11}$ Thus, the ET-induced muscular effects have not been directly compared with RT in a randomized controlled trial design.

The present study explored the intrinsic quadriceps muscle characteristics after ET compared with RT in COPD patients. We hypothesized that the two training modalities would counteract muscle dysfunction by different mechanisms: 1) ET by increasing oxidative muscle fiber proportion, capillarization, and oxidative capacity; and 2) RT by increasing muscle fiber cross-sectional area, glycolytic fiber proportion, and anaerobic capacity. By obtaining biopsies from the vastus lateralis muscle before and after 8 weeks of exercise training, we tested this hypothesis in 30 patients with moderate-to-severe COPD randomized to either ET or RT. Walking distance, maximal cycling endurance, and symptom burden were assessed to determine whether there were differences in training effects between groups.

Vascular function plays an important role in regulation of blood supply to working skeletal muscles, ${ }^{12}$ and vascular function may be impaired in patients with COPD, ${ }^{13}$ but whether exercise training alters blood flow to the working muscle in COPD has not been evaluated. Thus, our secondary aim was to evaluate the effects of training on leg muscle blood flow during exercise and vascular function determined by flow-mediated dilation (FMD).

\section{Methods}

This was a randomized controlled trial (1:1 allocation ratio) of ET compared with RT in patients with COPD. Eligibility criteria for participants were forced expiratory volume in 1 second/forced vital capacity ratio $<0.7$, forced expiratory volume in 1 second $<80 \%$ of predicted, Modified Medical Research Council score $\geq 2$, resting arterial oxygenation $>90 \%$, and age between 40 and 80 years. Exclusion criteria were claudication, severe heart failure, unstable ischemic heart disease, and malignant diseases. Spirometry (Model 2120; Vitalograph Ltd., Buckingham, UK) and a general medical examination were performed prior to inclusion. A computer-generated random allocation sequence was performed, and sequentially numbered opaque sealed envelopes were given to the participants after inclusion and baseline tests. The researcher who enrolled participants and analyzed data was blinded to the training intervention. Sample size was determined using 6-minute walking distance of $54 \mathrm{~m}$ (standard deviation [SD] of $50 \mathrm{~m}$ ) as the true difference (power $80 \%$ ) between groups with type 1 error risk of $5 \% .{ }^{14}$

The trial was approved by the Ethics Committee of the Copenhagen Region (H-2-2013-150), registered on
ClinicalTrials.gov (NCT02050945), and conducted in accordance with the guidelines of the Declaration of Helsinki. Verbal and written informed consent was obtained from all subjects before enrollment.

\section{Interventions}

Both groups of patients were trained for 35 minutes, 3 times a week for 8 weeks. The choice of the duration and frequency of the training sessions was based on comparable studies of ET versus RT in COPD. ${ }^{6}$ All training sessions were supervised to ensure safety, compliance, and progression of training intensity. Participants performed a short warm-up before and a cool-down after each training session.

ET was performed at moderate intensity adjusted individually to level 14-15 on the Borg scale of perceived exertion. The training sessions included either cycling on an ergometer or walking on a treadmill. The workload (Watts and distance) for each session was registered, and participants were instructed to increase exercise intensity progressively.

RT was performed on machines (Technogym, Cesena, Italy) and consisted of 4 sets of strength exercises of major upper and lower body muscle groups (chest press, rowing, leg press, and leg extension). The load was initially set at $30 \%$ of one repetition maximum and increased to $40 \%$ of one repetition maximum. Each exercise included 4 sets with a duration of 30 seconds that allowed for 15-20 repetitions to be completed. There was a 20 -second break between sets and a 60-second break between exercises. Subjects were instructed to maintain muscle tension at all times during sets. Workload (kilograms) was registered for all sessions, and intensity increased accordingly. The balance between set duration and rest allowed for muscular fatigue to be induced with a moderate load. As load was adjusted regularly to keep sets within the targeted repletion range, the relative training intensity was kept uniform among subjects.

\section{Outcomes}

\section{Symptom burden, exercise capacity, and body composition}

The symptom burden was assessed with the COPD assessment test which is a patient-completed questionnaire validated to monitor the impact of COPD on health-related quality of life (ranging from 0 to 40) where higher scores denote more severe impact. Exercise capacity was determined by a 6-minute walking test, peak oxygen uptake $\left(\mathrm{VO}_{2}\right.$ peak), and maximal workload $\left(W_{\max }\right)$. After verbal instruction, the 6-minute walking test was performed between 2 marked points $30 \mathrm{~m}$ apart. The $\mathrm{VO}_{2}$ peak was 
assessed (CPET System; Cosmed, Rome, Italy) during an incremental bicycle ergometer exercise test that included 5 minutes of unloaded pedaling followed by incremental steps of $10 \mathrm{~W}$ per minute until exhaustion $\left(W_{\max }\right)$. The subject's limit of tolerance was used as the criterion for finishing the test. The greatest 20 -second averaged $\mathrm{VO}_{2}$ was taken as the $\mathrm{VO}_{2}$ peak. Body composition was measured by whole-body dual-energy X-ray absorptiometry scan (Lunar Prodigy Advance; GE Healthcare, Madison, WI, USA).

\section{Muscle biopsies}

After local anesthesia (lidocaine 20\%) of the skin and muscle fascia, skeletal muscle biopsies were obtained by the Bergström technique $^{15}$ from the vastus lateralis of the nondominant leg. Biopsies were separated; one part for protein analysis was immediately frozen in liquid nitrogen, and another part for histology was mounted in Tissue-Tek ${ }^{\circledR}$ (Sakura Finetek USA, Inc., Torrance, CA, USA) and then immediately frozen in isopentane precooled in liquid nitrogen.

\section{Fiber type and capillarization}

Immunohistochemistry was performed using antibodies against myosin heavy chain (MHC) type I and type IIa, endothelial cells, and laminin. Muscle fibers positive for antibodies against both fiber types I and IIa were considered intermediate. Fibers of no coloration were deemed type IIx, and we subsequently verified this assumption with specific staining. The researcher who processed and analyzed the biopsies was blinded toward intervention and training status (antibodies and detailed description are given in the Supplementary materials).

\section{Muscle protein content}

We measured protein content of CS, eNOS, PFK, and VEGF by Western blotting. The housekeeping protein GAPDH was used as loading control (antibodies and detailed description are given in the Supplementary materials).

\section{Vascular ultrasound assessments}

We assessed endothelium-dependent dilation of the superficial femoral artery with FMD ${ }^{16}$ In brief, the arterial diameter was assessed by ultrasound at baseline for 2 minutes followed by 5-minute suprasystolic cuff inflation ( $220 \mathrm{mmHg})$. FMD was calculated as a percentage change from baseline in peak diameter after deflation (Supplementary materials). After the FMD, the participants were placed in a one-leg kicking chair. Leg blood flow (LBF) in the common femoral artery was measured using Doppler ultrasound. LBF was measured at rest and during 1 minute of passive leg movements followed by 3 -minute leg kicking at $12 \mathrm{~W} \cdot{ }^{17,18}$

\section{Statistical methods}

Effects within training groups were analyzed using a paired $t$-test, and differences between groups with Student's $t$-test comparing the mean change from baseline, but where the assumption of normality was not met, we used the Wilcoxon and Mann-Whitney $U$-tests. LBF measured at different time points was analyzed using a two-way analysis of variance with repeated measures (Holm-Sidak post hoc). Data were analyzed using SigmaPlot (v 13; Systat Software, San Jose, CA, USA). Results are presented as mean values (SD) unless mentioned otherwise. A two-tailed $P$-value $<0.05$ was considered statistically significant.

\section{Results}

Recruitment, training, and follow-up of participants were performed from February 2014 to March 2015 (Figure 1). The baseline characteristics of the participants are shown in Table 1.

The mean COPD assessment test score was reduced (less is better) after training in both the ET group (from 13 [SD 5] to 11 [SD 5], $P<0.05$ ) and the RT group (from 13 [SD 4] to 10 [SD 4], $P<0.05)$, with no significant differences between groups $(P>0.05)$. Six-minute walking distance and workload at $\mathrm{VO}_{2}$ peak increased in both training groups, also with no difference between groups $(P>0.05)$ (Table 2$)$.

\section{Quadriceps muscle morphology and capillarization}

A total of 18,301 muscle fibers were identified (mean of 482 [SD 222] fibers per biopsy). Representative transverse sections of muscle morphology before and after the two training modalities are shown in Figure 2A and B. We found no significant change in mean (SD) proportion of type I fibers with either of the training modalities (ET: from 34\% [SD 10] to $36 \%$ [SD 9]; RT: from 39\% [SD 12] to 36\% [SD 14]), and there were no significant differences between groups (Figure 2C). In contrast, the mean (SD) proportion of type IIa fibers decreased after ET (from $48 \%$ [SD 11] to $42 \%$ [SD 10], $P=0.022$ ) but did not change significantly after RT (from 48\% [SD 14] to 56\% [SD 16], $P>0.05$ ) with significant difference between groups $(P=0.040)$ (Figure 2D). The mean (SD) proportion of intermediate fibers (ET: from 13\% [SD 6] to 15\% [SD 7]; RT: from 9\% [SD 5] to 8\% [SD 6]) and type IIx fibers (ET: from 5\% [SD 5] to 7\% [SD 9]; RT: from median 2\% [range $0-6$ ] to median 1\% [range 0-2]) did not change significantly after training, with no difference 


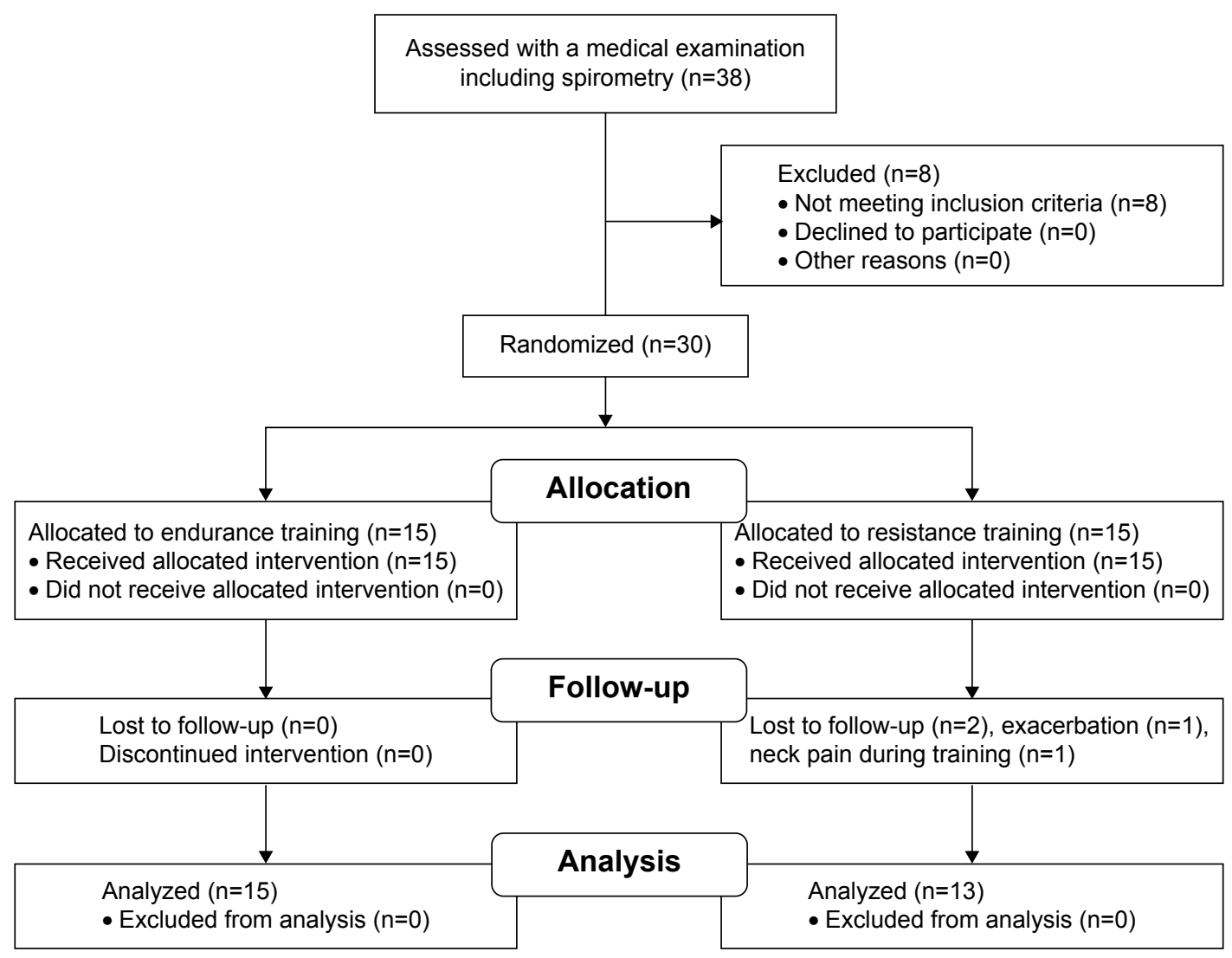

Figure I Study flow diagram.

between groups. We found no training effects within or between groups on capillarization (expressed as capillary-tofiber ratio and capillary density) or fiber cross-sectional area (Table 2).

Table I Baseline characteristics

\begin{tabular}{|c|c|c|c|}
\hline & $\begin{array}{c}\text { RT group } \\
(n=15)\end{array}$ & $\begin{array}{c}\text { ET group } \\
(n=15)\end{array}$ & $\begin{array}{c}\text { All participants } \\
(\mathrm{n}=\mathbf{3 0})\end{array}$ \\
\hline Age (years) & $65(7)$ & $60(9)$ & $63(8)$ \\
\hline Male, $\mathrm{n}$ & $6(40 \%)$ & 7 (47\%) & $13(43 \%)$ \\
\hline $\mathrm{FEV}_{1}, \%$ of predicted & $57(12)$ & $55(17)$ & $56(14)$ \\
\hline $\mathrm{FEV}_{1}(\mathrm{~L})$ & $1.5(0.5)$ & $\mathrm{I} .7(0.7)$ & $1.6(0.6)$ \\
\hline FVC (L) & $2.9(1.0)$ & $3.5(1.1)$ & $3.2(1.1)$ \\
\hline FVC, \% & $81(25)$ & $95(27)$ & $88(26)$ \\
\hline $\mathrm{FEV}_{1} / \mathrm{FVC}$ ratio & $0.56(0.2)$ & $0.5 I(0.1)$ & $0.54(0.1)$ \\
\hline$\geq 2$ exacerbations/year, $n$ & I (7\%) & $3(20 \%)$ & $4(13 \%)$ \\
\hline Pack-years & $42(10)$ & $31(16)$ & $36(14)$ \\
\hline Current smoker, $\mathrm{n}$ & $4(27 \%)$ & $3(20 \%)$ & $7(47 \%)$ \\
\hline BMI $\left(\mathrm{kg} / \mathrm{m}^{2}\right)$ & $27(4)$ & $27(6)$ & $27(5)$ \\
\hline Systolic BP (mmHg) & $139(17)$ & 137 (27) & $138(22)$ \\
\hline Diastolic BP (mmHg) & $86(10)$ & 91 (19) & $89(15)$ \\
\hline Diagnosed hypertension, $\mathrm{n}$ & $4(27 \%)$ & $8(53 \%)$ & $12(40 \%)$ \\
\hline Ischemic heart disease, $\mathrm{n}$ & I (7\%) & $2(13 \%)$ & $3(10 \%)$ \\
\hline Diabetes, $\mathrm{n}$ & I (7\%) & $2(13 \%)$ & $3(10 \%)$ \\
\hline
\end{tabular}

Note: Data in mean values (standard deviation) or numbers (\%).

Abbreviations: BMI, body mass index; BP, blood pressure; ET, endurance training; $\mathrm{FEV}_{1}$, forced expiratory volume in I second; FVC, forced vital capacity; $\mathrm{RT}$, resistance training.

\section{Muscle protein content}

Representative Western blots of CS and PFK are shown in Figure 3. ET tended to increase the content of CS $(P=0.08)$, and there was a reduction in PFK content $(P=0.049)$. In the RT group, there was no change in CS or PFK content $(P>0.05)$. Yet, we observed no difference between groups in muscle content of CS or PFK after training $(P>0.05)$. We found no training effects or differences between the groups in protein content of VEGF or eNOS $(P>0.05)$.

\section{Exercise hyperemia and vascular function}

There was no statistically significant difference in LBF within or between the two training groups $(P>0.05)$ (Figure $4 \mathrm{~A}$ and $\mathrm{B}$ ). When data from all participants were pooled, we found a higher LBF during $12 \mathrm{~W}$ of leg kicking after the exercise training $(P=0.03)$ (Figure 4C). Vascular function, assessed by \%FMD and LBF during passive movements of the lower leg, was similar before and after exercise training, and no differences between groups were found $(P>0.05)$ (Table 2).

\section{Discussion}

This randomized controlled trial of quadriceps muscle adaptations to ET versus RT in patients with COPD found that although both training modalities improved symptom burden, 
Table 2 Symptom burden, exercise capacity, body composition, vascular function, and capillarization

\begin{tabular}{|c|c|c|c|c|}
\hline & \multicolumn{2}{|c|}{ ET group } & \multicolumn{2}{|c|}{ RT group } \\
\hline & $\begin{array}{c}\text { Baseline } \\
\text { (mean [SD]) }\end{array}$ & $\begin{array}{l}\text { After training } \\
\text { (mean [SD]) }\end{array}$ & $\begin{array}{c}\text { Baseline } \\
\text { (mean [SD]) }\end{array}$ & $\begin{array}{l}\text { After training } \\
\text { (mean [SD]) }\end{array}$ \\
\hline CAT score & $13(5)$ & II (5) & $13(4)$ & $10(4)^{\#}$ \\
\hline 6MWD (m) & $550(80)$ & $589(55)^{\#}$ & $531(77)$ & $555(67)^{\#}$ \\
\hline Maximal workload (W) & $104(3 \mathrm{I})$ & $116(38)^{\#}$ & $95(40)$ & $104(4 I)^{\#}$ \\
\hline $\mathrm{VO}_{2}$ peak $(\mathrm{L} / \mathrm{min})$ & $1.6(0.5)$ & I.7 (0.5) & I.6 (0.6) & $1.7(0.6)$ \\
\hline $\mathrm{VO}_{2}$ peak $(\mathrm{mL} / \mathrm{kg} / \mathrm{min})$ & $21(7)$ & $22(7)$ & $21(6)$ & $23(7)$ \\
\hline RF max (breath/min) & $36(5)$ & $37(7)$ & $39(7)$ & $39(8)$ \\
\hline Tidal volume $\max (\mathrm{L})$ & $1.6(0.5)$ & $1.6(0.4)$ & $1.5(0.4)$ & $1.6(0.5)$ \\
\hline Minute ventilation $\max (\mathrm{L} / \mathrm{min})$ & $57.1(17.2)$ & $58.1(14.9)$ & $57.8(18.6)$ & $60.0(20.8)$ \\
\hline Total fat mass $(\mathrm{kg})$ & $25.3(9.4)$ & $24.1(9.4)^{\#}$ & $26.8(7.8)$ & $26.3(7.5)$ \\
\hline Total fat-free mass $(\mathrm{kg})$ & $52.1(10.3)$ & $52.3(10.7)$ & $49.5(10.6)$ & $49.7(I 1.0)$ \\
\hline Total lean mass $(\mathrm{kg})$ & $49.3(9.8)$ & $49.5(10.2)$ & $46.8(10.0)$ & $47.1(10.4)$ \\
\hline LBF, rest (L/min) & $0.16(0.07)$ & $0.17(0.07)$ & $0.19(0.08)$ & $0.16(0.05)$ \\
\hline LBF, 30 seconds passive (L/min) & $0.26(0.14)$ & $0.31(0.24)$ & $0.35(0.17)$ & $0.36(0.18)$ \\
\hline LBF, 60 seconds passive $(\mathrm{L} / \mathrm{min})$ & $0.28(0.16)$ & $0.35(0.27)$ & $0.4 I(0.19)$ & $0.4 I(0.22)$ \\
\hline FMD, \% & $5(3)$ & $5(3)$ & $7(6)$ & $6(6)$ \\
\hline Change in diameter $(\mathrm{mm})$ & $0.3(0.2)$ & $0.3(0.2)$ & $0.4(0.3)$ & $0.4(0.4)$ \\
\hline Time to peak (seconds) & $127(57)$ & $130(80)$ & $169(86)$ & $153(69)$ \\
\hline Capillary-to-fiber ratio & $1.529(0.534)$ & I.497 (0.547) & I.488 (0.279) & $\mathrm{I} .493(0.388)$ \\
\hline Capillaries per square millimeter & $275(87)$ & $250(94)$ & 24I (49) & $254(6 \mathrm{I})$ \\
\hline Cross-sectional area $\left(\mu \mathrm{m}^{2}\right)$ & $5,644(1,250)$ & $6,355(2,316)$ & $6,587(2,506)$ & $6,295(2,46 I)$ \\
\hline
\end{tabular}

Notes: "Difference within training group (paired $t$-test): $P<0.05$. No differences between groups (Student's $t$-test of mean change from baseline).

Abbreviations: 6MWD, 6-minute walking distance; CAT, COPD assessment test; ET, endurance training; FMD, flow-mediated dilation; LBF, leg blood flow; RF, respiration frequency; RT, resistance training; SD, standard deviation; $\mathrm{VO}_{2}$, oxygen uptake.

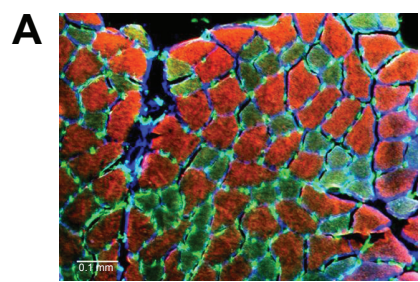

Before ET

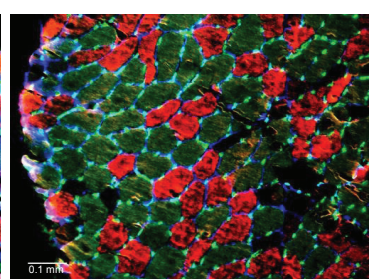

After ET

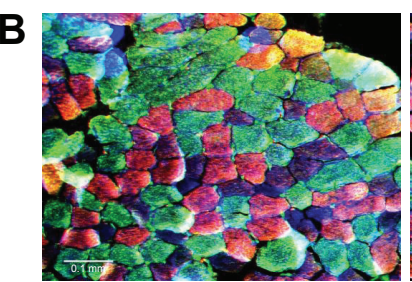

Before RT

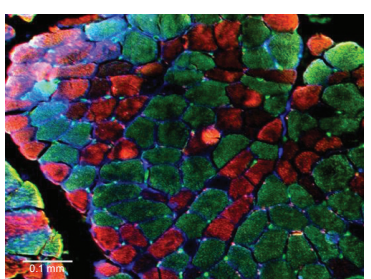

After RT
C

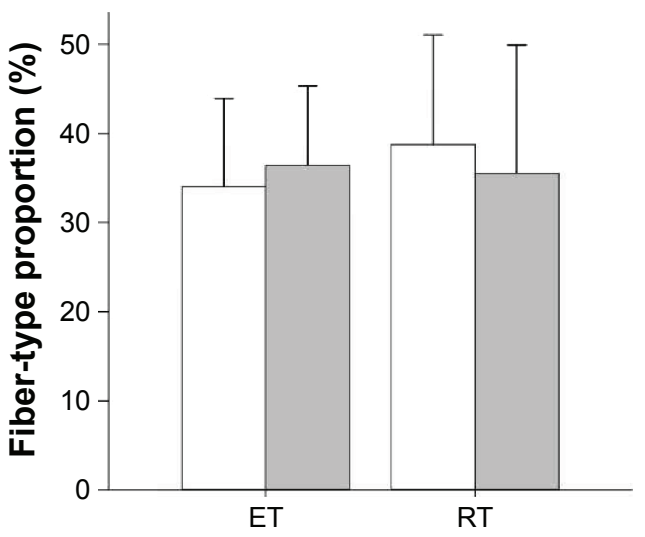

D

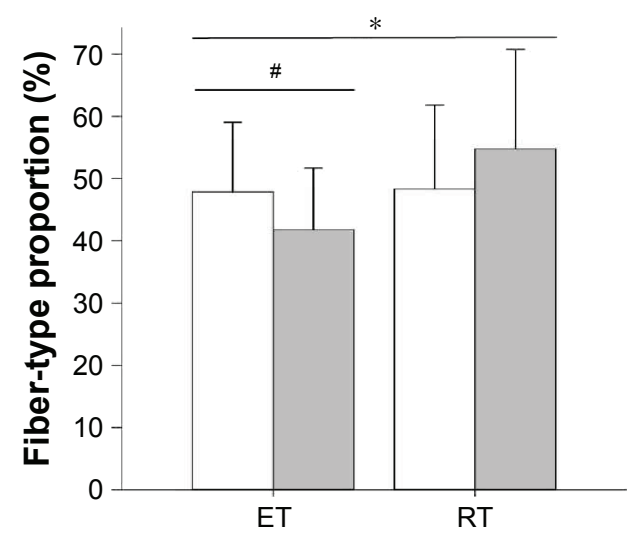

Before training $\square$ After training

Figure 2 Fiber-type change.

Notes: (A and B) Representative skeletal muscle cross-sections after immunohistochemistry were performed using antibodies against myosin heavy chain (MHC) type I (green), MHC lla (red) seen under a light microscope $\times 10$ objective. White scale bars $=100 \mu \mathrm{m}$. Muscle sections from two participants before and after (A) ET and (B) RT. (C and D) Mean values of fiber-type proportions (\%) with error bars (SD) in the ET group and the RT group. \#Significant difference within training group (paired $t$-test): $P<0.05$, *significant difference between groups (Student's $t$-test of mean change from baseline): $P<0.05$.

Abbreviations: ET, endurance training; MHC, myosin heavy chain; RT, resistance training. 
A ET (\#1) $\quad$ ET (\#2) $\quad$ ET (\#3) $\quad$ ET (\#4) $\quad$ RT (\#5) $\quad$ RT (\#6)

PFK

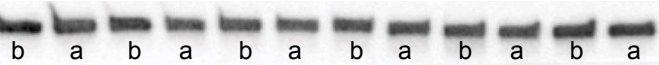

GAPDH

B ET (\#1) ET (\#2) ET (\#3) ET (\#4) RT (\#5) RT (\#6)

CS

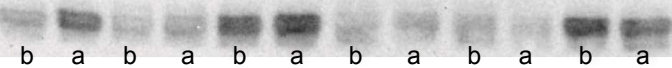

GAPDH

Figure 3 Muscle protein content.

Notes: Representative Western blots of (A) PFK, (B) CS, and GAPDH protein content in skeletal muscle from six COPD participants (\#I-6). Samples from the same participant were loaded side by side before (indicated as b) and after (indicated as a) ET and RT. Abbreviations: CS, citrate synthase; ET, endurance training; PFK, phosphofructokinase; RT, resistance training.

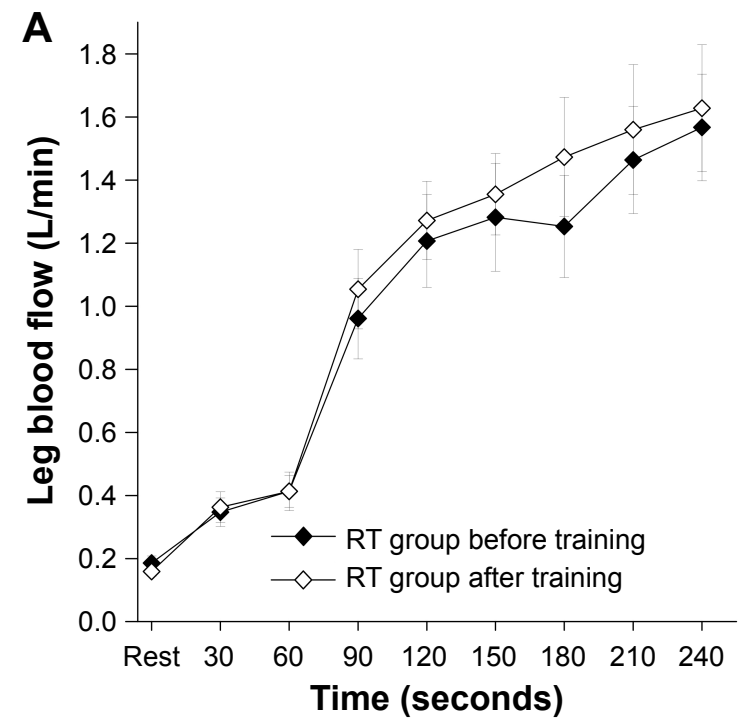

walking distance and maximal work load equally, leg muscles adapted differently. The proportion of glycolytic fibers was reduced after ET but not after RT. Likewise, ET induced a change in muscle protein content towards less glycolytic and more oxidative metabolism which was not observed after RT. Combined, the results indicate that both ET and RT induce functional improvements whereas ET appears more effective in shifting the quadriceps muscle towards a oxidative phenotype.

\section{Symptom burden and exercise capacity}

After 8 weeks of exercise training, the symptom burden was reduced to the same extent in both groups. Similarly, the effects on 6-minute walking distance and maximal cycling

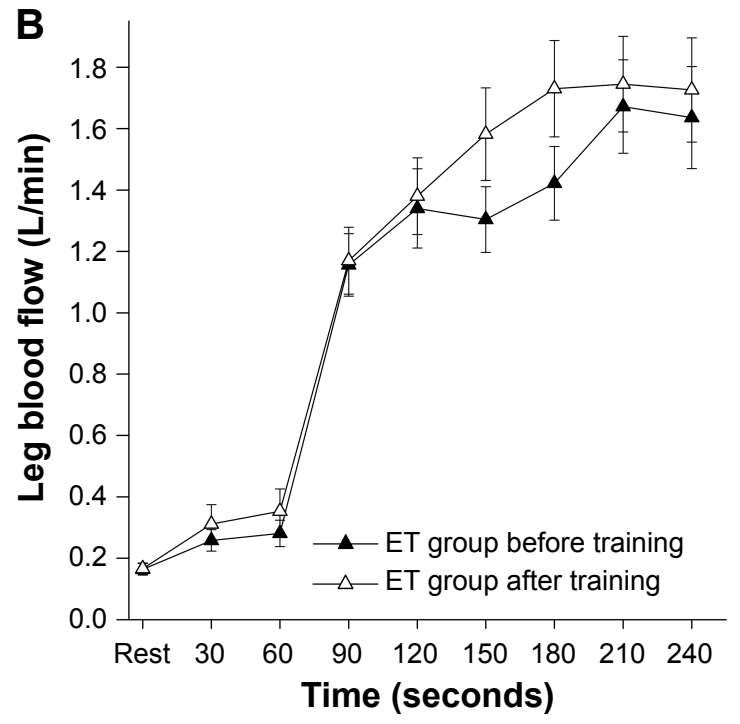

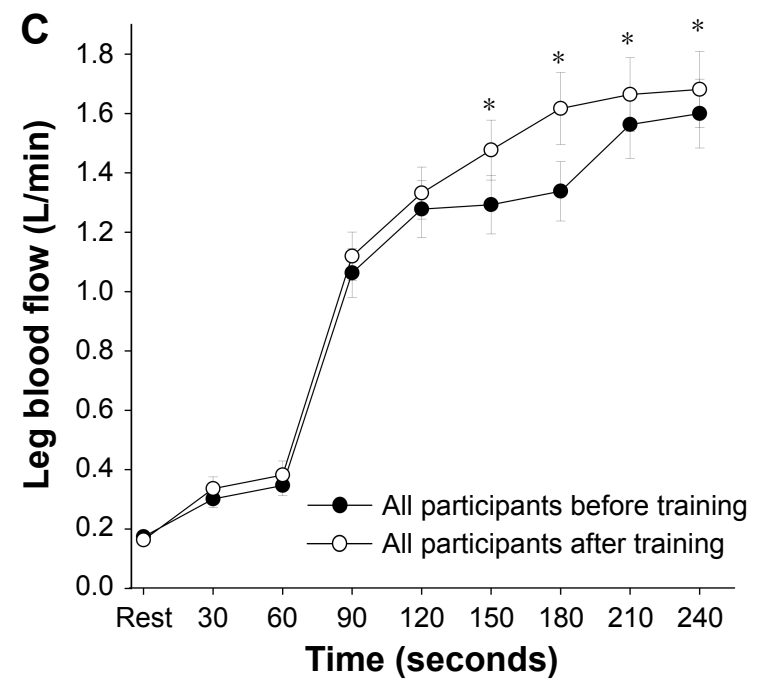

Figure 4 Leg muscle blood flow.

Notes: Leg blood flow (L/min) during I minute of passive leg movements followed by 3 minutes of leg kicking (I2 W) in (A) the RT group, (B) the ET group, and (C) all subjects before and after the training intervention. Data are presented as mean \pm standard error of mean. *Different from before training: $P<0.05$ (two-way ANOVA with repeated measures and Holm-Sidak post hoc).

Abbreviations: $\mathrm{ET}$, endurance training; $\mathrm{RT}$, resistance training. 
workload were similar in both groups. This study confirms that improved functional outcome after short-term exercise training can be achieved with both ET and RT. ${ }^{6,19}$ Although we did not find a statistically significant improvement of $\mathrm{VO}_{2}$ peak within groups, our results (increase by $\sim 0.1 \mathrm{LO}_{2} / \mathrm{min}$ ) were in alignment with previous studies of RT and ET in COPD. ${ }^{6}$ With a larger sample size, we find it likely that within-group changes of $\mathrm{VO}_{2}$ peak would have reached statistical significance.

\section{Skeletal muscle plasticity in COPD}

Skeletal muscle plasticity represents the ability of the muscle cell to alter the amount of protein and/or the type of protein in response to a given stimulus. ${ }^{20}$ When comparing the exercise-induced adaptations of muscle fiber type, we found a difference between the two training groups in type IIa fiber distribution. Thus, the type IIa fiber proportion decreased after ET, but in accordance with results by Lewis et al, ${ }^{9}$ we did not observe a change in IIa fiber proportion after RT. A quadriceps muscle fiber-type shift from oxidative type I fibers to glycolytic type II fibers is a consistent finding in COPD and is related to disease severity. ${ }^{21}$ Based on studies of the muscular adaptations to exercise in COPD compared to healthy age-matched subjects, it seems that RT could increase cross-sectional area ${ }^{22}$ and muscle mass, ${ }^{4}$ whereas ET appears to increase muscle oxidative capacity. ${ }^{10,11,23}$ In the present study, the proportion of type I fibers at baseline was low compared to reference values established in a healthy elderly population, ${ }^{24}$ and although not as low as observed in severe COPD,${ }^{21}$ the results were similar to results from studies of milder staged COPD. ${ }^{25,26}$ Even though our results did not show an increase in type I fiber proportion after ET, there appears to be a potential for an adaptation toward a more oxidative muscle phenotype after aerobic training in COPD. ${ }^{3,25}$ In the transition from muscle fibers showing pure expression of MHCIIa proteins to pure type I fibers are intermediate (MHCI/MHCIIa) fibers that co-express both MHC isoforms. ${ }^{20,27} \mathrm{We}$ speculate that longer training adherence would induce a greater muscle fiber-remodeling response, possibly with recruitment of these fibers in transition.

In support of the alterations in MHC fiber type with training, we found that ET lowered the level of the rate-limiting glycolytic enzyme PFK and tended to increase muscle content of the mitochondrial oxidative enzyme CS. The CS content is thought to be more abundant in oxidative type I fibers than in the more glycolytic type IIa fibers and the highly glycolytic type IIx fibers. ${ }^{28}$ In agreement, CS is reduced in COPD compared to healthy individuals. ${ }^{29}$ Moreover, studies indicate that COPD patients have a higher reliance on glycolytic metabolism during exercise and that upregulation of glycolytic enzymes (including PFK) is related to overproduction of lactate in muscles compared to healthy individuals. ${ }^{30,31}$ Ultimately, high muscle lactate can contribute to premature fatigue and exercise termination, ${ }^{26,31}$ but lactate may also stimulate central fatigue through afferent signaling from the exercising limb. ${ }^{32}$ Improving the oxidative capacity of the muscle in COPD patients would therefore likely improve exercise capacity.

\section{Leg muscle blood flow and vascular function}

Working skeletal muscle is highly dependent on sufficient blood supply to match the increased demand for oxygen. ${ }^{33}$ Thus, impaired blood flow to muscles may aggravate muscle dysfunction in COPD. We found that during an acute bout of exercise, both training groups showed an initial raise and steady state in exercise hyperemia, as also observed in healthy individuals. ${ }^{34}$ There was an overall effect of training on exercise hyperemia, but the increase was not significant within each of the two groups separately. One explanation for the overall improvement in exercise hyperemia after training could be improved vascular function. ${ }^{12}$ Although previous evidence has indicated that the vascular function is impaired in COPD, ${ }^{35}$ others have showed no difference in vascular function ${ }^{36}$ and even higher LBF response to exercise in COPD compared with healthy control subjects. ${ }^{36,37}$ Compared to previously reported data in healthy elderly individuals, our data support that vascular function is impaired in COPD, as the FMD response was low. ${ }^{38}$ Low FMD indicates impaired nitric oxide (NO)mediated vasodilation, ${ }^{39}$ but NO is not an obligatory substance for exercise hyperemia. In the current study, vascular function was not improved by training as indicated by no change in either FMD or response to passive leg movement, another NOdependent test. ${ }^{18}$ Moreover, there was no change in quadriceps muscle protein content of eNOS after exercise training in either of the training groups. Another explanation for the increase in exercise hyperemia could be a reduced vasoconstriction after training. The ability to override sympathetic vasoconstrictor activity during exercise, termed functional sympatholysis, appears to impair leg muscle blood flow in patients with essential hypertension, while exercise training improves exercise hyperemia and functional sympatholysis. ${ }^{17,40}$

\section{Limitations}

This study is explorative, and there are some limitations that should be considered. Because established thresholds of 
minimal clinically important difference for muscle outcomes in COPD do not exist, our sample size calculation was not based on muscle outcome. We did not obtain sufficient muscle specimens from all subjects. Consequently, the paired number of samples from both pre- and post-training was smaller in the RT group than in the ET group for structural measures $(n=6$ versus $n=13$ ) and similarly for the analysis of muscle protein content ( $n=9$ versus $n=14$ ). Maybe, the difference between the two training forms regarding effect on intrinsic muscle function is caused by that the quadriceps muscle was stimulated throughout all ET training sessions, but only during approximately $50 \%$ of each RT training session. Because of the small sample size and explorative nature of this study, we did not adjust for possible confounding variables including the age and the level of pulmonary function. Finally, we used perceived exertion to adjust the intensity of ET, but the actual exercise intensity may have varied between subjects depending on pulmonary function.

\section{Conclusion and clinical implications}

This study confirms that ET and RT improve symptoms and exercise capacity in patients with COPD. However, our data suggest that ET induces a more oxidative quadriceps muscle phenotype compared with RT. These results indicate that aerobic physical activity (eg, cycling or walking) at moderate intensity is the most efficient countermeasure of quadriceps muscle dysfunction in COPD, and we suggest that training of patients with COPD should include an aerobic component.

\section{Acknowledgments}

Karina Olsen, Hanne Villumsen, Ruth Rovsing, Noemi G James, and Jacob Marott are acknowledged for their technical assistance. The Centre for Physical Activity Research (CFAS) is supported by a grant from TrygFonden. During the study period, the Centre of Inflammation and Metabolism (CIM) was supported by a grant from the Danish National Research Foundation (DNRF55). The study was further supported by grants from the Axel Muusfeldts Foundation, the Capital Region of Denmark, and the Novo Nordisk Foundation. CIM/CFAS is a member of DD2 - the Danish Center for Strategic Research in Type 2 Diabetes (the Danish Council for Strategic Research, grant nos 09-067009 and 09-075724).

\section{Author contributions}

All authors contributed toward data analysis, drafting and critically revising the paper and agree to be accountable for all aspects of the work.

\section{Disclosure}

The authors report no conflicts of interest in this work.

\section{References}

1. Maltais F, Decramer M, Casaburi R, et al. An official American Thoracic Society/European Respiratory Society statement: update on limb muscle dysfunction in chronic obstructive pulmonary disease. Am J Respir Crit Care Med. 2014;189(9):e15-e62.

2. McCarthy B, Casey D, Devane D, Murphy K, Murphy E, Lacasse Y. Pulmonary rehabilitation for chronic obstructive pulmonary disease. Cochrane Database Syst Rev. 2015;2:CD003793.

3. Vogiatzis I, Terzis G, Stratakos G, et al. Effect of pulmonary rehabilitation on peripheral muscle fiber remodeling in patients with COPD in GOLD stages II to IV. Chest. 2011;140(3):744-752.

4. Constantin D, Menon MK, Houchen-Wolloff L, et al. Skeletal muscle molecular responses to resistance training and dietary supplementation in COPD. Thorax. 2013;68(7):625-633.

5. Bolton CE, Bevan-Smith EF, Blakey JD, et al. British Thoracic Society guideline on pulmonary rehabilitation in adults. Thorax. 2013;68 (Suppl 2):ii1-ii30.

6. Iepsen UW, Jorgensen KJ, Ringbaek T, Hansen H, Skrubbeltrang C, Lange $\mathrm{P}$. A systematic review of resistance training versus endurance training in COPD. J Cardiopulm Rehabil Prev. 2015;35(3):163-172.

7. Chodzko-Zajko WJ, Proctor DN, Fiatarone Singh MA, et al. American College of Sports Medicine position stand. Exercise and physical activity for older adults. Med Sci Sports Exerc. 2009;41(7):1510-1530.

8. Slot IG, van den Borst B, Hellwig VA, Barreiro E, Schols AM, Gosker HR. The muscle oxidative regulatory response to acute exercise is not impaired in less advanced COPD despite a decreased oxidative phenotype. PLoS One. 2014;9(2):e90150.

9. Lewis MI, Fournier M, Storer TW, et al. Skeletal muscle adaptations to testosterone and resistance training in men with COPD.J Appl Physiol (1985). 2007;103(4):1299-1310.

10. Maltais F, LeBlanc P, Simard C, et al. Skeletal muscle adaptation to endurance training in patients with chronic obstructive pulmonary disease. Am J Respir Crit Care Med. 1996;154(2 Pt 1):442-447.

11. Vogiatzis I, Simoes DC, Stratakos G, et al. Effect of pulmonary rehabilitation on muscle remodelling in cachectic patients with COPD. Eur Respir J. 2010;36(2):301-310.

12. Mortensen SP, Saltin B. Regulation of the skeletal muscle blood flow in humans. Exp Physiol. 2014;99(12):1552-1558.

13. Maclay JD, McAllisterDA, Mills NL, et al. Vascular dysfunction in chronic obstructive pulmonary disease. Am J Respir Crit Care Med. 2009;180(6): 513-520.

14. Dupont WD, Plummer WD Jr. Power and sample size calculations. A review and computer program. Control Clin Trials. 1990;11(2): 116-128.

15. Bergström J. Percutaneous needle biopsy of skeletal muscle in physiological and clinical research. Scand J Clin Lab Invest. 1975;35(7): 609-616.

16. Harris RA, Nishiyama SK, Wray DW, Richardson RS. Ultrasound assessment of flow-mediated dilation. Hypertension. 2010;55(5): $1075-1085$.

17. Mortensen SP, Nyberg M, Gliemann L, Thaning P, Saltin B, Hellsten Y. Exercise training modulates functional sympatholysis and $\alpha$-adrenergic vasoconstrictor responsiveness in hypertensive and normotensive individuals. J Physiol. 2014;592(14):3063-3073.

18. Mortensen SP, Askew CD, Walker M, Nyberg M, Hellsten Y. The hyperaemic response to passive leg movement is dependent on nitric oxide: a new tool to evaluate endothelial nitric oxide function. J Physiol. 2012;590(17):4391-4400.

19. Iepsen UW, Jørgensen KJ, Ringbæk T, Hansen H, Skrubbeltrang C, Lange $P$. A combination of resistance and endurance training increases leg muscle strength in COPD: an evidence-based recommendation based on systematic review with meta-analyses. Chron Respir Dis. 2015; 12(2):132-145. 
20. Pette D. Skeletal muscle plasticity - history, facts and concepts. In: Bottinelli R, Reggiani C, editors. Skeletal Muscle Plasticity in Health and Disease: From Genes to Whole Muscle. Dordrecht: Springer; 2006.

21. Gosker HR, Zeegers MP, Wouters EF, Schols AM. Muscle fibre type shifting in the vastus lateralis of patients with COPD is associated with disease severity: a systematic review and meta-analysis. Thorax. 2007;62(11):944-949.

22. Menon MK, Houchen L, Singh SJ, Morgan MD, Bradding P, Steiner MC. Inflammatory and satellite cells in the quadriceps of patients with COPD and response to resistance training. Chest. 2012;142(5): 1134-1142.

23. Puente-Maestu L, Tena T, Trascasa C, et al. Training improves muscle oxidative capacity and oxygenation recovery kinetics in patients with chronic obstructive pulmonary disease. Eur J Appl Physiol. 2003;88(6): 580-587.

24. Gouzi F, Maury J, Molinari N, et al. Reference values for vastus lateralis fiber size and type in healthy subjects over 40 years old: a systematic review and metaanalysis. J Appl Physiol (1985). 2013;115(3): 346-354.

25. Gouzi F, Préfaut C, Abdellaoui A, et al. Blunted muscle angiogenic training response in COPD patients versus sedentary controls. Eur Respir J. 2013;41(4):806-814.

26. van den Borst B, Slot IG, Hellwig VA, et al. Loss of quadriceps muscle oxidative phenotype and decreased endurance in patients with mild-tomoderate COPD. J Appl Physiol (1985). 2013;114(9):1319-1328.

27. Andersen JL. Muscle fibre type adaptation in the elderly human muscle. Scand J Med Sci Sports. 2003;13(1):40-47.

28. Galpin AJ, Raue U, Jemiolo B, Trappe TA, Harber MP, Trappe S. Human skeletal muscle fiber type specific protein content. Anal Biochem. 2012;425(2):175-182.

29. Maltais F, LeBlanc P, Whittom F, et al. Oxidative enzyme activities of the vastus lateralis muscle and the functional status in patients with COPD. Thorax. 2000;55(10):848-853.

30. Allaire J, Maltais F, Doyon JF, et al. Peripheral muscle endurance and the oxidative profile of the quadriceps in patients with COPD. Thorax. 2004;59(8):673-678.
31. Saey D, Lemire BB, Gagnon P, et al. Quadriceps metabolism during constant workrate cycling exercise in chronic obstructive pulmonary disease. J Appl Physiol (1985). 2011;110(1):116-124.

32. Gagnon $P$, Bussières JS, Ribeiro F, et al. Influences of spinal anesthesia on exercise tolerance in patients with chronic obstructive pulmonary disease. Am J Respir Crit Care Med. 2012;186(7):606-615.

33. Andersen P, Saltin B. Maximal perfusion of skeletal muscle in man. J Physiol. 1985;366:233-249.

34. Maltais F, Jobin J, Sullivan MJ, et al. Metabolic and hemodynamic responses of lower limb during exercise in patients with COPD. $J$ Appl Physiol (1985). 1998;84(5):1573-1580.

35. Eickhoff P, Valipour A, Kiss D, et al. Determinants of systemic vascular function in patients with stable chronic obstructive pulmonary disease. Am J Respir Crit Care Med. 2008;178(12):1211-1218.

36. Hartmann SE, Waltz X, Leigh R, Anderson TJ, Poulin MJ. Blood flow during handgrip exercise in COPD: effect of vitamin C. Med Sci Sports Exerc. 2016;48(2):200-209.

37. Richardson RS, Leek BT, Gavin TP, et al. Reduced mechanical efficiency in chronic obstructive pulmonary disease but normal peak VO2 with small muscle mass exercise. Am J Respir Crit Care Med. 2004;169(1):89-96.

38. Shechter M, Shechter A, Koren-Morag N. Usefulness of brachial artery flow-mediated dilation to predict long-term cardiovascular events in subjects without heart disease. Am J Cardiol. 2014;113(1): $162-167$.

39. Corretti MC, Anderson TJ, Benjamin EJ, et al. Guidelines for the ultrasound assessment of endothelial-dependent flow-mediated vasodilation of the brachial artery: a report of the International Brachial Artery Reactivity Task Force. J Am Coll Cardiol. 2002;39(2):257-265.

40. Hellsten Y, Jensen L, Thaning P, Nyberg M, Mortensen S. Impaired formation of vasodilators in peripheral tissue in essential hypertension is normalized by exercise training: role of adenosine and prostacyclin. J Hypertens. 2012;30(10):2007-2014. 


\section{Supplementary materials Methods}

\section{Vascular ultrasound}

We assessed endothelium-dependent dilation of the superficial femoral artery with flow-mediated dilation (FMD). We used an ultrasound machine (Logic E9; GE Healthcare, Milwaukee, WI, USA) equipped with a probe operating at an imaging frequency of $9 \mathrm{MHz}$. Longitudinal ultrasound images of the artery were optimized in B-mode and saved in DICOM files until analysis. FMD was measured by the same sonographer at approximately the same time of the day before and after training, prior to 15-20 minutes of supine rest. A cuff (D. E. Hokanson Inc., Belevue, WA, USA) was placed $\sim 10 \mathrm{~cm}$ above the knee, and all recordings of vessel diameter were made proximally of the cuff. The arterial diameter was assessed at baseline for 2 minutes followed by 5 -minute suprasystolic cuff inflation $(\sim 220 \mathrm{mmHg})$. Vessel diameter recordings were resumed from $\sim 10$ seconds before deflation and the following 5 minutes. The FMD was calculated as a percent change from baseline in peak diameter after deflation. Analysis of the artery diameter was performed using validated software (Vascular Research Tool v. 6; Medical Imaging Applications LLC., Coralville, IA, USA) for automated border detection of the saved DICOM files.

Shortly after, the FMD subjects were placed in a oneleg kicking chair. Leg blood flow in the common femoral artery was measured using Doppler ultrasound (frequency of 4.3-5.0 MHz). All recordings were obtained at the lowest possible insonation angle and always $<60^{\circ}$. The sample volume was maximized according to the width of the vessel and kept clear of the vessel walls. Doppler traces and B-mode images were recorded continuously, and Doppler traces were averaged over minimum eight heart cycles. Arterial diameter was measured during the systole and two-thirds of the diameter during the diastole. Leg blood flow was measured at baseline and during 1 minute of passive leg movements followed by 3-minute active leg kicking at $12 \mathrm{~W}$.

\section{Immunohistochemistry}

Directly after performing the muscle biopsy from the vastus lateralis muscle, the biopsies were prepared using Tissue-Tek ${ }^{\circledR}$ O.C.T. ${ }^{\text {TM }}$ Compound (Sakura Finetek USA, Inc., Torrance, CA, USA). The biopsies were frozen immediately and kept at $-80^{\circ} \mathrm{C}$.

The muscle biopsies were cut into 8-10 $\mu \mathrm{m}$-thin cross sections at $-30^{\circ} \mathrm{C}$ using a cryostat (Microm HM500 M; Heidelberg, Germany), and the sections were then placed onto microscopy glass slides. The specimens were kept at $-20^{\circ} \mathrm{C}$ until all the biopsies were cut and ready for immunohistochemistry.

The cross sections were then fixed for 2 minutes using a formaldehyde solution (F1635; Sigma-Aldrich Co., Sigma, MO, USA). Following rinsing the sections with a $\mathrm{pH}$ neutral 1\% phosphate-buffered saline (PBS) solution, the specimens were blocked for 10 minutes using a $1 \%$ bovine serum albumin (A7906; Sigma-Aldrich Co.). The primary antibodies (myosin heavy chain [MHC] type I [M2421; Sigma-Aldrich Co.] and MHCIIa [SC-71; Developmental Studies Hybridoma Bank, Iowa, IA, USA]) were diluted in an antibody diluent (S0809; Dako Denmark A/S, Glostrup, Denmark) together with polyclonal rabbit anti-laminin (Z0097; Dako Denmark A/S), and the sections incubated for 50 minutes. Following a rinse with PBS solution, the cross sections were incubated with the secondary antibodies for 50 minutes. The incubations were performed at room temperature.

The capillaries were identified using Biotinylated Ulex Europaeus Agglutinin I (B-1065; Vector Laboratories, Burlingame, CA, USA), which marks endothelial cells. The applied secondary antibodies were the following: donkey anti-mouse $\operatorname{IgG}(\mathrm{H}+\mathrm{L})$ secondary antibody, Alexa Fluor ${ }^{\circledR}$ 555 conjugate (A31570; Thermo Fisher Scientific, Waltham, MA, USA) and streptavidin conjugated with fluorescein isothiocyanate (F0422; Dako Denmark A/S); donkey anti-mouse IgG $(\mathrm{H}+\mathrm{L})$ secondary antibody, Alexa Fluor ${ }^{\circledR} 488$ conjugate (A21202; Thermo Fisher Scientific); and goat anti-rabbit $\operatorname{IgG}(\mathrm{H}+\mathrm{L})$ secondary antibody, Alexa Fluor ${ }^{\circledR} 350$ conjugate (A21068; Thermo Fisher Scientific).

The cross sections were mounted using Prolong Gold Antifade Mountant (P36930; Life Technologies) and were stored at $-20^{\circ} \mathrm{C}$ until pictures of the biopsies were taken by a high-resolution camera (CoolSNAP; Photometrics, Tucson, AZ, USA) through a light microscope (Axioplan 2 Imaging; Carl Zeiss Meditec AG, Jena, Germany). The muscle fibers and capillaries were counted using both computer programs, Microsoft PowerPoint (Microsoft Corporation, Redmond, WA, USA) and ImageJ 1.47 (National Institutes of Health, Bethesda, MD, USA).

Overlay pictures of the different immunofluorescent images taken were used when assessing the muscle fiber distribution of the biopsies, as type I fibers were recognized as those expressing MHCI, type IIa fibers as those expressing MHCIIa, and the intermediate fibers as those expressing both MHCI and MHCIIa.

Type IIx fibers were identified as those fibers lacking expression of both primary antibodies, MHCI and MHCIIa. 
Staining the biopsies with a specific MHCIIx antibody (6H1; Developmental Studies Hybridoma Bank) validated this assumption.

\section{Western blotting}

Muscle specimens were cut at $-20^{\circ} \mathrm{C}$, and samples were homogenized and lysed. The protein content was measured (colorimetric assay; Bio-Rad Laboratories Inc., Hercules, CA, USA) to insure equal sample concentrations. Loading buffer (Thermo Fisher Scientific) and dithiothreitol were applied, and samples were boiled for 5 minutes. The lysate was loaded onto the gels (Criterion TGX 4\%-15\% gel; Bio-Rad Laboratories Inc.) where samples from one individual (before and after training) were loaded side by side on the same gel. Gels were run at $105 \mathrm{~V}$ for $\sim 60$ minutes. Protein was then transferred onto a polyvinylidene fluoride membrane (Trans-Blot Turbo; Bio-Rad Laboratories Inc.). Membranes were blocked and incubated with primary antibodies overnight. Membranes were washed and incubated with secondary horseradish peroxidase-conjugated IgG antibody (Dako Denmark A/S). Signal was detected (Supersignal West Femto Luminal/Enhancer Solution; Thermo Fisher Scientific) and exposed (charge couple camera; Bio-Rad Laboratories Inc.). Blots were analyzed and quantified using Image Lab software (Bio-Rad Laboratories Inc.).

Primary antibodies used were the following:

1. CS (rabbit polyclonal antibody), Abcam (Cambridge, UK; cat\# 96600)

2. eNOS (rabbit monoclonal antibody), Cell Signaling Technology (Danvers, MA, USA; cat\# 9586)

3. GAPDH (rabbit monoclonal antibody), Cell Signaling Technology (cat\# 2118)

4. PFK (mouse monoclonal antibody), R\&D Systems, Inc. (Minneapolis, MN, USA; cat\# MAB7687)

5. VEGF (rabbit monoclonal antibody A-20), Santa Cruz Biotechnology Inc. (Dallas, TX, USA; cat\# SC152).
International Journal of COPD

\section{Publish your work in this journal}

The International Journal of COPD is an international, peer-reviewed journal of therapeutics and pharmacology focusing on concise rapid reporting of clinical studies and reviews in COPD. Special focus is given to the pathophysiological processes underlying the disease, intervention programs, patient focused education, and self management protocols.

\section{Dovepress}

This journal is indexed on PubMed Central, MedLine and CAS. The manuscript management system is completely online and includes a very quick and fair peer-review system, which is all easy to use. Visit http://www.dovepress.com/testimonials.php to read real quotes from published authors. 